\title{
INFLUENCIA DE LA ZONA DE ORIGEN EN LA COMPRA DE HORTALIZAS EN LA IX REGIÓN DE CHILE ${ }^{1}$
}

\author{
INFLUENCES OF THE ZONE OF ORIGIN ON THE PURCHASE \\ OF VEGETABLES IN IX REGION OF CHILE
}

\author{
Berta Schnettler Morales ${ }^{2}$; Cristián Zavala Ruiz ${ }^{2}$; Rodolfo Pihán Soriano ${ }^{2}$
}

\begin{abstract}
RESUMEN
Considerando la importancia del consumo de hortalizas para el cuidado de la salud de los consumidores, se determinó la frecuencia de consumo y gasto semanal en hortalizas, el grado de conocimiento sobre sus características nutricionales y de producción y la importancia de la zona de origen del producto en la decisión de compra en Temuco, mediante una encuesta personal a 241 consumidores. Se obtuvo que la mayoría consume hortalizas diariamente y gasta entre $\$ 4.000$ y $\$ 7.000$ semanales, presentando un grado medio de conocimiento sobre las hortalizas. Mediante análisis conjunto se determinó en tomate que en general la variedad fue más importante que el precio y la zona de origen en la decisión de compra. Los consumidores prefirieron la variedad de tomate corriente producida en Angol y privilegian pagar precios bajos.
\end{abstract}

Palabras clave: consumo de hortalizas, zona de origen, análisis conjunto.

\begin{abstract}
Considering the relevance of the vegetables consumption for consumers' health care, it was determined the frequency of consumption and weekly cost in vegetables, knowledge about its nutritious and production characteristics, and the importance of the zone of origin of the product in the purchase decision in Temuco, through a personal survey to 241 consumers. It was obtained that most of the people consume vegetables daily and spend between $\$ 4,000$ and $\$ 7,000$ Chilean pesos weeky, presenting a medium degree of knowledge about vegetables. By means of conjoint analysis it was determined that in the case of tomato, in general the variety was more important than the price and the zone of origin in the purchase decision. Consumers preferred the simple tomato variety produced in Angol and they privileged to pay low prices.
\end{abstract}

Key words: vegetables consumption, zone of origin, conjoint analysis.

\section{INTRODUCCIÓN}

La sociedad actual se caracteriza por un aumento de la conciencia por el cuidado de la salud y un creciente interés por el rol de los alimentos en el mejoramiento del bienestar y salud de los consumidores (Gilbert, 2000). En la actualidad, en la mayoría de los países occidentales se recomienda un elevado consumo de frutas y hortalizas, consideradas beneficiosas para la salud (Nicklaus et al., 2004). Numerosa evidencia sugiere que el consumo regular de frutas y hortalizas ayuda a prevenir enfermedades cardiovasculares, ciertos tipos de cáncer (WHO, 2002) y otras enfermedades degenerativas crónicas (IFIC, 2001). La Organización Mundial de la Salud sugiere un consumo diario de $400 \mathrm{~g}$ de frutas y hortalizas (WHO Study Group, 1990). En respuesta a lo anterior, durante los 90 se lanzaron numerosas campañas de promoción del consumo de hortalizas y frutas (Cox et al., 1996). Sin embargo, un reciente reporte de WHO/ FAO (2003) señala que el consumo diario total de frutas y hortalizas se encuentra $100 \mathrm{~g}$ por debajo de lo recomendado.

La imagen de la zona de origen de un alimento se ha usado activamente, para promocionar las

1 Financiado por la Universidad de La Frontera, Proyecto DIUFRO No 120601.

2 Universidad de La Frontera, Departamento de Producción Agropecuaria, Facultad de Ciencias Agropecuarias y Forestales, Casilla 54-D, Temuco, Chile. E-mail: bschnett@ufro.cl 
características superiores de calidad asociadas a la experiencia humana y condiciones naturales de la zona de origen. La imagen de la zona de origen da una identidad única al producto y genera valor agregado (Van Ittersum et al., 2003). La influencia del origen en la evaluación del consumidor ha sido ampliamente estudiada desde la perspectiva del país de origen (Agrawald y Kamakura, 1999; Verlegh y Steenkamp, 1999; Orth y Firbasová, 2003; Bernués et al., 2003; Schnettler et al., 2004; Balestrini y Gamble, 2006, entre otros), respecto a que los consumidores diferencian entre productos de diferentes países de origen. Siendo esperable que el consumidor diferencie entre distintas zonas de origen como regiones, provincias o comunas (Balling, 1995), las investigaciones al respecto son escasas (Van Ittersum et al., 2003). En Chile, sólo en 2005 entraron en vigencia las modificaciones realizadas a la Ley 19.039 de Propiedad Industrial, que incluyen la implementación de un sistema de registro para indicaciones geográficas y denominaciones de origen, en respuesta a la oportunidad para ampliar mercados, ocupando nichos con alto nivel de exigencia en calidad (ODEPA, 2006).

La alimentación y bebida concentra la mayor proporción del gasto de los hogares chilenos (27\% en el total de hogares). Dentro de esta agrupación la mayor proporción se destina al gasto en bebidas y restaurantes $(23,9 \%)$, seguido por carne $(18,4 \%)$ y pan, cereales y pastas $(17,4 \%)$, mientras el gasto en hortalizas y tubérculos alcanza a 10,1\% (INE, 1997). La producción hortícola nacional se concentra en la zona central del país. Entre las regiones $\mathrm{V}$ y VII se concentra $68,7 \%$ de la superficie destinada a este rubro, destacando la Región Metropolitana con 23\%. La IX Región representa 4\% de la superficie nacional de hortalizas, de la cual sólo $35,7 \%$ se destina a cultivo comercial. La comuna de Angol concentra $6,9 \%$ de la superficie hortícola regional, ocupando el quinto lugar de importancia luego de Nueva Imperial, Padre Las Casas, Temuco y Freire (INE, 1998), pero destaca por la reconocida calidad de los "tomates angolinos".

En base a estos antecedentes, el objetivo del presente estudio fue determinar variables del consumo de hortalizas, el grado de conocimiento de aspectos nutricionales y de producción, la importancia de la zona de origen en la compra y diferencias en la aceptación y disposición a pagar por hortalizas producidas en distintas zonas de Chile, según características demográficas y de comportamiento de compra de los consumidores de la ciudad de Temuco, IX Región de Chile.

\section{MATERIALES Y MÉTODOS}

Se realizó una encuesta personal a una muestra de 241 consumidores de la ciudad de Temuco, cuyo número se obtuvo mediante la fórmula de muestreo probabilístico irrestricto aleatorio según la población de Temuco al Censo de 2002 (245.347 habitantes. INE, 2003), considerando $90 \%$ de confianza y $5 \%$ de error de estimación con p y q de 0,5 (Scheaffer et al., 1996). Como instrumento de recogida de información se utilizó un cuestionario con preguntas cerradas sobre la frecuencia de compra y gasto semanal en la compra de hortalizas para el hogar, además de preguntas de clasificación de los encuestados: género, edad, zona de residencia, número de integrantes del grupo familiar; ocupación y estudios del jefe de hogar y la tenencia de 10 bienes domésticos; estas dos últimas variables para determinar el grupo socioeconómico según Adimark (2004). La encuesta se aplicó en dos supermercados de Temuco entre agosto y octubre de 2006, posterior a la validación del cuestionario mediante un pretest con el $20 \%$ de la muestra.

Se incluyó una pregunta cuyo objetivo fue medir cuán correcto era el conocimiento de los encuestados sobre aspectos nutricionales y de la producción de hortalizas, consistente en ocho frases de verdadero o falso (Las hortalizas son una importante fuente de vitaminas. Las hortalizas producidas en la zona sur no tienen contaminación microbiológica. Las hortalizas presentadas en bolsas de plástico y rotuladas no tienen contaminación. Las hortalizas hidropónicas están libres de todo tipo de contaminación. Algunas hortalizas contienen antioxidantes. Las hortalizas orgánicas son tratadas con agroquímicos. Las hortalizas aportan fibra a la dieta. Algunas hortalizas poseen una acción depurativa en el organismo). Para cada frase los encuestados debieron contestar verdadero o falso y cuán seguros estaban de la respuesta usando una escala de siete puntos ( $7=$ absolutamente seguro, $1=$ completamente inseguro). Basado en estas respuestas se calculó el grado de conocimiento según la siguiente ecuación (Verdume y Viane, 2003):

$$
G C=\left\{\left[\sum_{i=l}^{5}\left(A_{i} x C_{i}\right)\right] / 8\right\} x 100
$$

donde $\mathrm{GC}=$ grado de conocimiento $(\%) ; \mathrm{A}_{\mathrm{i}} \in\{0,1\}$; $\mathrm{C}_{\mathrm{i}} \in\{0,00 ; 0,17 ; 0,33 ; 0,50 ; 0,67 ; 0,83 ; 1,00\}$. Si 
la respuesta $(\mathrm{V}$ o $\mathrm{F})$ fue correcta/incorrecta se le asignó puntuación 1/0 $\left(\mathrm{A}_{\mathrm{i}}\right)$. El factor de certeza 0,00 se asignó si dentro de la escala de 7 puntos se eligió "uno"; 0,17 si se eligió "dos"; 0,33 si se eligió "tres"; 0,50 al elegir "cuatro"; 0,67 al seleccionar "cinco"; 0,83 al seleccionar "seis" y 1,00 si se eligió "siete". El GC se obtuvo dividiendo la suma de los ocho $\left(\mathrm{A}_{\mathrm{i}}\right.$ x $\left.\mathrm{C}_{\mathrm{i}}\right)$ por ocho y multiplicando este resultado por 100 .

Para determinar la importancia de la zona de origen de la hortaliza en la decisión de compra respecto a la variedad y precio en tomate, se utilizó análisis conjunto (AC) que corresponde a una técnica multivariante basada en la premisa de que los consumidores evalúan el valor de un producto/servicio/ idea (real o hipotética) combinando cantidades separadas de valor que proporciona cada atributo. Dada la valoración que un conjunto de individuos hace de determinadas configuraciones alternativas de producto, se puede deducir la importancia de los atributos que configuran estas alternativas y de sus diferentes niveles (Hair et al. 1999). Los niveles definidos para la variedad fueron larga vida y corriente; en zona de origen se definió zona central y Angol y en precio $\$ 150 / \mathrm{kg}$ y $\$ 300 / \mathrm{kg}$, correspondientes a precios de mercado durante la época estival. A partir de estos atributos y niveles se obtuvieron 8 combinaciones $(2 \times 2 \times 2)$ identificadas con una letra desde la A hasta la H: A) tomate larga vida producido en la zona central a $\$ 150 / \mathrm{kg}$, B) tomate larga vida producido en Angol a $\$ 150 / \mathrm{kg}$, C) tomate larga vida producido en la zona central a $\$ 300 / \mathrm{kg}$, D) tomate larga vida producido en Angol a $\$ 300 / \mathrm{kg}$, E) tomate corriente producido en la zona central a $\$ 150 / \mathrm{kg}$, F) tomate corriente producido en Angol a $\$ 150 / \mathrm{kg}$, $\mathrm{G})$ tomate corriente producido en la zona central a $\$ 300 / \mathrm{kg}, \mathrm{H}$ ) tomate corriente producido en Angol a \$300/kg. La función de preferencia correspondió al Modelo de Punto Ideal. Para la recogida de datos se usó el procedimiento de perfil total, para lo cual se elaboraron ocho tarjetas con una especificación para cada atributo. A los encuestados se les solicitó que ordenaran las tarjetas desde la más preferida hasta la menos preferida usando una escala de 1 a 8 (1 más preferida; 8 menos preferida). Para el atributo precio se estableció una relación lineal, debido a que generalmente a menor precio la utilidad o preferencia es mayor. Los atributos restantes fueron considerados como variables discretas. Para determinar la bondad de ajuste del modelo conjunto se utilizó correlación de Pearson y se usó la Tau de Kendall para determinar si la ordenación de los estímulos estimada según la función de ordenación corresponde con la ordenación real del encuestado (Hair et al., 1999). Basado en los resultados del AC se estimó la disposición a pagar marginal (DAP-Mg) por hortalizas producidas en las dos zonas de origen usadas. De acuerdo con Gan y Luzar (1993), la DAP-Mg se estima por medio del cuociente negativo entre el coeficiente (valor parcial de utilidad) de cada nivel de un atributo y el coeficiente del atributo precio (valor $\int>\beta$ ). El procedimiento de cálculo es el siguiente:

$$
D A P-M g=-\left(\frac{\text { Utilidad } \text { origen }}{\beta \text { precio }}\right)
$$

Los resultados de las preguntas de frecuencia de consumo y gasto semanal en hortalizas fueron analizados mediante Tablas de Contingencia según las características demográficas, geográficas y de compra de los encuestados y el estadístico Chi Cuadrado, que permite inferir si dos o más magnitudes de frecuencias de casos de la población pueden ser consideradas similares (Levin y Rubin, 1996). Se usó SPSS 14.0 (SPSS.Inc., USA) para Windows, que para el caso del análisis conjunto presenta el modelo de estimación de parámetros correspondiente a MANOVA.

\section{RESULTADOS Y DISCUSIÓN}

La muestra quedó compuesta por 29,0\% hombres y $71,0 \%$ mujeres; $18,6 \%$ personas entre 20 y 34 años, $47,4 \%$ entre 35 y 49 años y $34,0 \%$ de 50 años y más; $95 \%$ de residencia urbana y $5 \%$ en zonas rurales; $13,3 \%$ trabajadores por cuenta propia, $10,4 \%$ empresarios, 35,3\% empleados particulares, 17,0\% empleados públicos, $22,0 \%$ jubilados, $0,8 \%$ cesantes y $1,2 \%$ en otra situación laboral; $17,0 \%$ de familias con uno o dos integrantes, $54,7 \%$ con tres o cuatro integrantes y $28,3 \%$ con cinco o más integrantes; $51,8 \%$ perteneciente al grupo socioeconómico (GSE) $\mathrm{ABC} 1$ (alto y medio-alto), 39,0\% a C2 (medio-medio), $7,1 \%$ a C3 (medio-bajo) y 2,1 a D (bajo).

Frecuencia de consumo y gasto en hortalizas. En el Cuadro 1 se observa que en la muestra total la mayor proporción de encuestados declaró consumir hortalizas en forma diaria $(66,0 \%)$, seguido por una frecuencia de consumo de tres veces por semana 


\section{Cuadro 1}

Frecuencia semanal de consumo y gasto en hortalizas en el hogar, según características demográficas y geográficas del consumidor. Temuco, octubre de 2006

\begin{tabular}{|c|c|c|c|c|c|c|c|c|}
\hline \multirow{2}{*}{ Muestra } & \multicolumn{4}{|c|}{ Frecuencia de consumo } & \multicolumn{4}{|c|}{ Gasto (\$) } \\
\hline & Diaria & $\begin{array}{c}3 \text { veces } \\
\text { por sem. }\end{array}$ & $\begin{array}{l}2 \text { veces } \\
\text { por sem }\end{array}$ & Otra & 0-3.999 & 4.000-7.000 & $7.001-15.000$ & $>15.000$ \\
\hline Total\% & 66,0 & 23,2 & 4,6 & 6,2 & 15,4 & 49,4 & 33,2 & 2,1 \\
\hline \multicolumn{5}{|c|}{ Género\% $P=0,071$} & \multicolumn{4}{|c|}{$P=0,183$} \\
\hline Femenino & 70,2 & 20,5 & 2,9 & 6,4 & 12,3 & 52,0 & 33,9 & 1,8 \\
\hline Masculino & 55,7 & 30,0 & 8,6 & 5,7 & 22,9 & 42,9 & 31,4 & 2,9 \\
\hline \multicolumn{5}{|l|}{ GSE $P=0,079$} & \multicolumn{4}{|c|}{$P=0,000$} \\
\hline $\mathrm{ABC} 1$ & 75,2 & 18,4 & 3,2 & 3,2 & 12,0 & 39,2 & 44,8 & 4,0 \\
\hline $\mathrm{C} 2$ & 58,5 & 25,5 & 6,4 & 9,6 & 17,0 & 61,7 & 21,3 & 0 \\
\hline $\mathrm{C} 3$ & 47,1 & 35,3 & 5,9 & 11,8 & 17,6 & 64,7 & 17,6 & 0 \\
\hline $\mathrm{D}$ & 40,0 & 60,0 & 0 & 0 & 60,0 & 20,0 & 20,0 & 0 \\
\hline \multicolumn{5}{|c|}{ Grupo etario $P=0,124$} & \multicolumn{4}{|c|}{$P=0,000$} \\
\hline 20-34 años & 53,3 & 28,9 & 11,1 & 6,7 & 40,0 & 51,1 & 6,7 & 2,2 \\
\hline 35-49 años & 71,1 & 18,4 & 4,4 & 6,1 & 8,8 & 50,0 & 37,7 & 3,5 \\
\hline 50 años o más & 65,9 & 26,8 & 1,2 & 6,1 & 11,0 & 47,6 & 41,5 & 0 \\
\hline \multicolumn{5}{|c|}{ Grupo familiar $P=0,094$} & \multicolumn{4}{|c|}{$P=0,000$} \\
\hline 1-2 integrantes & 51,2 & 29,3 & 9,8 & 9,8 & 26,8 & 51,2 & 22,0 & 0 \\
\hline 3-4 integrantes & 67,4 & 25,8 & 3,0 & 3,8 & 16,7 & 54,5 & 25,8 & 3,0 \\
\hline 5 ó más & 72,1 & 14,7 & 4,4 & 8,8 & 5,9 & 38,2 & 54,4 & 1,5 \\
\hline \multicolumn{5}{|l|}{ Zona $P=0,004$} & \multicolumn{4}{|c|}{$P=0,283$} \\
\hline Urbana & 65,9 & 24,0 & 3,5 & 6,6 & 14,8 & 50,2 & 33,2 & 1,7 \\
\hline Rural & 66,7 & 8,3 & 25,0 & 0 & 25,0 & 33,3 & 33,3 & 8,3 \\
\hline \multicolumn{5}{|c|}{ Ocupación $P=0,462$} & \multicolumn{4}{|c|}{$P=0,076$} \\
\hline Cuenta propia & 59,4 & 21,9 & 9,4 & 9,4 & 6,3 & 59,4 & 34,4 & 0 \\
\hline Empresario & 80,0 & 16,0 & 0 & 4,0 & 0 & 44,0 & 56,0 & 0 \\
\hline Emp. Particular & 67,1 & 24,7 & 3,5 & 4,7 & 23,5 & 40,0 & 34,1 & 2,4 \\
\hline Emp. Público & 65,9 & 22,0 & 7,3 & 4,9 & 22,0 & 53,7 & 24,4 & 0 \\
\hline Jubilado & 64,2 & 26,4 & 3,8 & 5,7 & 11,3 & 54,7 & 28,3 & 5,7 \\
\hline Busca trabajo & 50,0 & 0 & 0 & 50,0 & 0 & 100 & 0 & 0 \\
\hline Otra situación & 33,3 & 33,3 & 0 & 33,3 & 0 & 66,7 & 33,3 & 0 \\
\hline \multicolumn{5}{|c|}{ Frecuencia de compra } & \multicolumn{4}{|c|}{$P=0,000$} \\
\hline \multirow{4}{*}{\multicolumn{5}{|c|}{$\begin{array}{l}\text { Diaria } \\
\text { Tres veces por semana } \\
\text { Dos veces por semana } \\
\text { Otra }\end{array}$}} & 8,8 & 44,4 & 44,7 & 2,5 \\
\hline & & & & & 28,6 & 58,9 & 10,7 & 1,8 \\
\hline & & & & & 45,5 & 54,5 & 0 & 0 \\
\hline & & & & & 13,3 & 66,7 & 20,0 & 0 \\
\hline
\end{tabular}

GSE: Grupo socioeconómico.

$(23,2 \%)$. De estos resultados se desprende que $34 \%$ de los encuestados se encuentra por debajo de las recomendaciones de consumo diario de hortalizas de la Organización Mundial de la Salud. Sólo se detectaron diferencias estadísticamente significativas $(\mathrm{p} \leq 0,05)$ según el lugar de residencia, debido a que una menor proporción de consumidores de zonas rurales consume hortalizas tres veces por semana 
y es mayor el porcentaje que lo hace dos veces por semana. Respecto al gasto semanal de los hogares en hortalizas, en la muestra total predominó un gasto entre $\$ 4.000$ y $\$ 7.000$ con $49,4 \%$ de las respuestas, seguido por un gasto entre $\$ 7.001$ y $\$ 15.000$ con $33,2 \%$ del total. Se obtuvieron diferencias estadísticas ( $\mathrm{p} \leq 0,001)$ según el grupo socioeconómico, edad del consumidor y tamaño de la familia, debido a la mayor proporción de personas de $\mathrm{ABC} 1$ que gasta semanalmente entre \$ 7.001 y \$ 15.000 , el elevado porcentaje de los grupos C2 y C3 que gasta entre $\$ 4.000$ y $\$ 7.000$ y la alta fracción del estrato de menores ingresos que gasta menos de $\$ 4.000$. Este resultado concuerda con lo obtenido por Leather (1995) en el Reino Unido, respecto a que los consumidores de menores ingresos gastan menos en hortalizas, sobre todo en las más caras. Es destacable que un nivel de gasto superior a $\$ 15.000 /$ semana sólo se detectó en el grupo ABC1. En el caso de los estratos etarios, las diferencias se explican por la alta proporción de personas jóvenes (20-34 años) que gasta menos de $\$ 4.000$ a la semana y la mayor proporción de personas de 50 años y más que gasta entre \$ 7.001 y \$ 15.000. Esto último coincide con el incremento de la preocupación por la salud que se produce con edades mayores, detectado por Verbeke et al. (2000). Según el tamaño de la familia, destaca la mayor proporción de encuestados de familias con cinco o más integrantes que gasta entre $\$ 7.001$ y $\$ 15.000$ y el superior porcentaje de familias con uno o dos integrantes que gasta menos de $\$ 4.000$. Al cruzar la frecuencia de consumo y el gasto semanal en hortalizas se observaron diferencias estadísticas ( $\mathrm{p} \leq 0,001)$, debido a la mayor proporción de encuestados que consume diariamente hortalizas que gasta entre \$ 7.001 y \$ 15.000 semanales, el superior porcentaje que consume hortalizas tres veces por semana cuyo gasto fluctúa entre $\$ 4.000$ y $\$ 7.000$ y la elevada fracción que consume hortalizas sólo dos veces por semana que gasta menos de $\$ 4.000$.

Conocimiento sobre características de las hortalizas. La determinación del grado de conocimiento (GC) sobre aspectos nutricionales y de producción de hortalizas a través de ocho frases de verdadero y falso sobre el tema arrojó un nivel medio correspondiente a un GC de $54,4 \%$ en la muestra total (Cuadro 2). Los GC obtenidos en las distintas submuestras presentaron pequeñas variaciones respecto a la media de la muestra total. Levemente por sobre este valor se encontraron los consumidores masculinos; personas pertenecientes al estrato $A B C 1$, entre 20 y 34 años y de 50 años y más, los de familias con menos de cinco miembros; residentes en zonas rurales; trabajadores por cuenta propia y jubilados. En la muestra total 3,5\% de los encuestados presentó un GC igual o menor a $25 \%$; $37,8 \%$ tuvo un GC entre 26 y $50 \%$; 51,5\% entre 51 y $75 \%$ y $7,1 \%$ obtuvo un GC entre 76 y $100 \%$. Sólo se obtuvieron diferencias estadísticamente significativas $(\mathrm{p} \leq 0,05)$ según el número de integrantes de la familia, debido al mayor porcentaje de encuestados pertenecientes a los hogares más numerosos que presentó un GC menor o igual a $25 \%$ y a la menor proporción del mismo grupo con GC entre 51 y $76 \%$. Las frases que registraron mayor proporción de respuestas correctas en la muestra total fueron "Las hortalizas son una fuente de vitaminas" (verdadera), "Las hortalizas aportan fibra a la dieta" (verdadera) y "Algunas hortalizas poseen una acción depurativa en el organismo" con cifras en superiores a 95\%. A continuación se encontraron "Las hortalizas orgánicas son tratadas con agroquímicos" (falsa) y "Las hortalizas producidas en la zona sur no tienen contaminación microbiológica" (falsa) con valores en torno a $70 \%$, seguidas por "Las hortalizas presentadas en bolsas de plástico y rotuladas no tienen contaminación" (falsa) y "Las hortalizas hidropónicas están libres de todo tipo de contaminación" (falsa) con cifras alrededor a $50 \%$. "Algunas hortalizas contienen antioxidantes" (verdadera) fue la pregunta que registró menor proporción de respuestas correctas con 10,0\%. Esto sugiere la necesidad de aumentar la difusión de las características nutricionales y de producción de las hortalizas entre los consumidores.

Importancia de la zona de origen en la decisión de compra. Como se observa en el Cuadro 3 , en la muestra total se obtuvo que el factor de mayor importancia en la compra de hortalizas fue la variedad $(42,7 \%)$, seguido por el precio $(32,9 \%)$ y la zona de origen $(24,2 \%)$. Este resultado se contrapone a lo determinado por Orth y Firbasová (2003), Balestrini y Gamble (2006) y Schnettler et al. (2004) en cuanto a la mayor importancia del origen en la decisión de compra, desde la perspectiva de distintos países de procedencia de los alimentos. Si bien este orden de importancia se mantuvo en la mayoría de las submuestras analizadas, destacaron los consumidores entre 20 y 34 años, residentes en zonas rurales y cesantes para quienes después 


\section{Cuadro 2}

Grado de conocimiento (GC) sobre aspectos nutricionales y de producción de hortalizas según características demográficas y geográficas de los consumidores. Temuco, octubre de 2006

\begin{tabular}{|c|c|c|c|c|c|}
\hline Muestra & Promedio (\%) & $0-25 \%$ & $26-50 \%$ & $51-75 \%$ & $76-100 \%$ \\
\hline Total & 54,4 & 3,7 & 37,8 & 51,5 & 7,1 \\
\hline Género & & \multicolumn{4}{|c|}{$P=0,159$} \\
\hline Femenino & 53,8 & 4,1 & 38,6 & 52,6 & 4,7 \\
\hline Masculino & 55,9 & 2,9 & 35,7 & 48,6 & 12,9 \\
\hline GSE & & \multicolumn{4}{|c|}{$P=0,583$} \\
\hline $\mathrm{ABC} 1$ & 56,1 & 4,0 & 32,8 & 52,8 & 10,4 \\
\hline $\mathrm{C} 2$ & 53,6 & 3,2 & 41,5 & 51,1 & 4,3 \\
\hline $\mathrm{C} 3$ & 47,6 & 5,9 & 47,1 & 47,1 & 0,0 \\
\hline $\mathrm{D}$ & 52,9 & 0,0 & 60,0 & 40,0 & 0,0 \\
\hline Grupo etario & & \multicolumn{4}{|c|}{$P=0,145$} \\
\hline 20-34 años & 56,5 & 6,7 & 31,1 & 46,7 & 15,6 \\
\hline 35-49 años & 53,0 & 4,4 & 40,4 & 50,0 & 5,3 \\
\hline 50 años o más & 55,4 & 1,2 & 37,8 & 56,1 & 4,9 \\
\hline Grupo familiar & & \multicolumn{4}{|c|}{$P=\mathbf{0 , 0 3 3}$} \\
\hline 1-2 integrantes & 58,5 & 2,4 & 31,7 & 56,1 & 9,8 \\
\hline 3-4 integrantes & 54,9 & 0,8 & 38,6 & 54,5 & 6,1 \\
\hline 5 o más integrantes & 51,0 & 10,3 & 39,7 & 42,6 & 7,4 \\
\hline Zona & & \multicolumn{4}{|c|}{$P=0,264$} \\
\hline Urbana & 54,3 & 3,5 & 38,9 & 51,1 & 6,6 \\
\hline Rural & 57,5 & 8,3 & 16,7 & 58,3 & 16,7 \\
\hline Ocupación & & \multicolumn{4}{|c|}{$P=0,096$} \\
\hline Cuenta propia & 57,6 & 3,1 & 25,0 & 62,5 & 9,4 \\
\hline Empresario & 52,4 & 12,0 & 40,0 & 44,0 & 4,0 \\
\hline Empleado particular & 54,3 & 1,2 & 43,5 & 47,1 & 8,2 \\
\hline Empleado público & 51,1 & 7,3 & 36,6 & 51,2 & 4,9 \\
\hline Jubilado & 56,9 & 0,0 & 35,8 & 56,6 & 7,5 \\
\hline Cesante & 44,7 & 0,0 & 100 & 0,0 & 0,0 \\
\hline Otra situación & 50,0 & 33,3 & 0,0 & 66,7 & 0,0 \\
\hline
\end{tabular}

de la variedad fue más relevante el origen que el precio en la decisión de compra. Asimismo, para los consumidores en "otra situación laboral" la variedad y el precio tuvieron igual importancia, mientras para los consumidores con "otra" frecuencia semanal de consumo la importancia de los tres atributos fue similar, el origen fue levemente más importante $(35,7 \%)$ que la variedad $(33,4 \%)$ y el precio $(30,9 \%)$, lo que indica que ninguno de los atributos domina las preferencias del consumidor. Totalmente opuesto fue el comportamiento de las personas que gastan más de $\$ 15.000$ semanales en hortalizas, para quienes la mayor importancia correspondió al precio, seguido por la variedad y el origen. En general, los coeficientes de correlación de Pearson y Tau de Kendall obtenidos en la muestra total y submuestras fueron cercanos a 1, lo que indica una buena bondad de ajuste del modelo conjunto y que la ordenación de los estímulos presentados en las tarjetas corresponde con la ordenación global del encuestado, siendo ambos estadísticamente significativos $(\mathrm{p} \leq 0,001)$. La excepción corresponde al grupo de personas en "otra" situación laboral que presentó coeficientes de Pearson y Tau de Kendall bajos y no significativos $(p>0,05)$. 


\section{Cuadro 3}

Importancia de los atributos variedad, zona y precio (\%) en el proceso de decisión de compra de tomate, según variables demográficas, geográficas y de compra de los consumidores. Temuco, octubre de 2006

\begin{tabular}{|c|c|c|c|c|c|c|}
\hline Muestra & Variedad & Origen & Precio & R Pearson & Tau Kendall & $\beta$ precio \\
\hline Total & 42,7 & 24,2 & 32,9 & 0,999 & 1,000 & $-1,861$ \\
\hline \multicolumn{7}{|l|}{ Género } \\
\hline Femenino & 41,8 & 24,3 & 33,9 & 0,999 & 1,000 & $-1,901$ \\
\hline Masculino & 43,6 & 24,4 & 32,0 & 1,000 & 1,000 & $-1,886$ \\
\hline \multicolumn{7}{|l|}{ GSE } \\
\hline $\mathrm{ABC} 1$ & 44,3 & 20,0 & 35,7 & 0,999 & 1,000 & $-2,020$ \\
\hline $\mathrm{C} 2$ & 40,4 & 29,5 & 30,1 & 1,000 & 1,000 & $-1,686$ \\
\hline $\mathrm{C} 3$ & 42,9 & 27,1 & 30,0 & 0,981 & 0,929 & $-1,588$ \\
\hline $\mathrm{D}$ & 49,5 & 20,1 & 30,4 & 0,998 & 1,000 & $-2,100$ \\
\hline \multicolumn{7}{|l|}{ Grupo etario } \\
\hline 20-34 años & 46,2 & 27,0 & 26,8 & 0,990 & 0,982 & $-1,433$ \\
\hline 35-49 años & 44,2 & 22,9 & 32,8 & 0,999 & 1,000 & $-1,892$ \\
\hline 50 años o más & 38,9 & 24,5 & 36,6 & 0,999 & 1,000 & $-2,140$ \\
\hline \multicolumn{7}{|l|}{ Grupo familiar } \\
\hline 1-2 integrantes & 47,2 & 22,9 & 29,9 & 0,996 & 0,929 & $-1,976$ \\
\hline 3-4 integrantes & 43,1 & 24,6 & 32,3 & 1,000 & 0,929 & $-1,864$ \\
\hline 5 o más int. & 39,4 & 24,4 & 36,2 & 0,996 & 0,929 & $-1,787$ \\
\hline \multicolumn{7}{|l|}{ Zona } \\
\hline Urbana & 43,0 & 24,4 & 32,6 & 0,999 & 1,000 & $-1,838$ \\
\hline Rural & 40,0 & 32,5 & 27,5 & 0,985 & 0,929 & $-1,125$ \\
\hline \multicolumn{7}{|l|}{ Ocupación } \\
\hline Cuenta propia & 35,3 & 30,5 & 34,2 & 0,975 & 0,929 & $-1,500$ \\
\hline Empresario & 41,5 & 22,3 & 36,2 & 0,996 & 0,929 & $-1,780$ \\
\hline Emp. particular & 44,5 & 23,3 & 32,2 & 0,998 & 1,000 & $-2,035$ \\
\hline Emp. público & 41,9 & 25,3 & 32,8 & 0,986 & 1,000 & $-1,854$ \\
\hline Jubilado & 45,9 & 20,5 & 33,6 & 0,999 & 1,000 & $-2,000$ \\
\hline Cesante & 50,0 & 40,0 & 10,0 & 1,000 & 1,000 & $-0,500$ \\
\hline Otra situación & 38,1 & 38,1 & 23,8 & 0,375 & 0,294 & 0,000 \\
\hline \multicolumn{7}{|c|}{ Frecuencia de compra } \\
\hline Diaria & 44,4 & 21,4 & 34,2 & 0,999 & 1,000 & $-1,978$ \\
\hline 3 veces/semana & 41,0 & 28,9 & 30,1 & 0,998 & 0,929 & $-1,598$ \\
\hline 2 veces/semana & 41,5 & 25,4 & 33,1 & 0,939 & 0,837 & $-1,909$ \\
\hline Otra frecuencia & 33,4 & 35,7 & 30,9 & 0,957 & 0,786 & $-1,567$ \\
\hline \multicolumn{7}{|l|}{ Gasto semanal } \\
\hline$\$ 0-3.999$ & 42,7 & 24,7 & 32,6 & 0,993 & 0,857 & $-2,081$ \\
\hline$\$ 4.000-7.000$ & 44,7 & 27,1 & 28,2 & 1,000 & 1,000 & $-1,513$ \\
\hline$\$ 7.001-15.000$ & 40,5 & 20,2 & 39,3 & 0,994 & 1,000 & $-2,250$ \\
\hline Más de $\$ 15.000$ & 34,2 & 16,6 & 49,2 & 0,982 & 0,929 & $-2,300$ \\
\hline
\end{tabular}

Considerando las variables de segmentación utilizadas, destacaron las personas del estrato D y los cesantes por la mayor importancia dada a la variedad con cifras cercanas a 50\%. La situación contraria se detectó en los consumidores que presentaron "otra" frecuencia semanal de compra. La más alta valoración del precio en la compra correspondió a las personas del estrato $\mathrm{ABC} 1$, de 
50 años y más, pertenecientes a familias con cinco o más integrantes, empresarios y personas que gastan entre $\$ 7.001$ y $\$ 15.000$ y más de $\$ 15.000$ a la semana en hortalizas, lo que concuerda con lo determinado por Baltas y Doyle (1998), respecto a que los consumidores que compran un alimento en cantidades altas son más sensibles al precio. Al respecto, es importante señalar que la proporción de empresarios con familias de cinco integrantes o más fue superior a la muestra total (48\%), detectándose diferencias estadísticamente significativas en esta variable $(p=0,004)$. Por el contrario, la menor importancia dada al precio correspondió a los cesantes y personas residentes en zonas rurales, lo que en este último grupo puede deberse al autoconsumo de hortalizas de producción propia. La zona de origen de la hortaliza fue de mayor relevancia para las personas residentes en zonas rurales, quienes presumiblemente por estar asociados a la producción agrícola perciben como amenaza la producción extrarregional; las personas con "otra" frecuencia de compra, "otra" situación laboral y los cesantes, debiendo indicarse que estos grupos fueron en su totalidad minoritarios dentro de la muestra analizada (iguales o inferiores a 6,2\%).

Utilidad de los atributos evaluados. Como se observa en el Cuadro 4, en la muestra total el

\section{Cuadro 4}

Utilidad de los niveles de variedad, zona de origen, precio de tomate y Disposición Marginal a Pagar (DAP-Mg) por la zona de origen según variables demográficas, geográficas y de compra de los consumidores. Temuco, octubre de 2006

\begin{tabular}{|c|c|c|c|c|c|c|c|c|}
\hline \multirow[t]{2}{*}{ Muestra } & \multicolumn{2}{|c|}{$\begin{array}{l}\text { Utilidad } \\
\text { Variedad }\end{array}$} & \multicolumn{2}{|c|}{$\begin{array}{c}\text { Utilidad } \\
\text { Zona de origen }\end{array}$} & \multicolumn{2}{|c|}{$\begin{array}{l}\text { Utilidad } \\
\text { Precio }\end{array}$} & \multicolumn{2}{|c|}{$\begin{array}{c}\text { DAP-Mg } \\
(\%)\end{array}$} \\
\hline & Larga vida & Corriente & Central & Angol & $\$ 150$ & $\$ 300$ & Central & Angol \\
\hline Total & $-0,656$ & 0,656 & $-0,600$ & 0,600 & $-1,861$ & $-3,722$ & 99,86 & 100,14 \\
\hline $\begin{array}{l}\text { Femenino } \\
\text { Masculino }\end{array}$ & $\begin{array}{l}-0,482 \\
-0,718\end{array}$ & $\begin{array}{l}0,482 \\
0,718\end{array}$ & $\begin{array}{l}-0,573 \\
-0,654\end{array}$ & $\begin{array}{l}0,573 \\
0,654\end{array}$ & $\begin{array}{l}-1,901 \\
-1,886\end{array}$ & $\begin{array}{l}-3,801 \\
-3,771\end{array}$ & $\begin{array}{l}99,87 \\
99,85\end{array}$ & $\begin{array}{l}100,13 \\
100,15\end{array}$ \\
\hline $\begin{array}{l}\mathrm{ABC} 1 \\
\mathrm{C} 2 \\
\mathrm{C} 3 \\
\mathrm{D}\end{array}$ & $\begin{array}{l}-0,620 \\
-0,644 \\
-0,912 \\
-0,900\end{array}$ & $\begin{array}{l}0,620 \\
0,644 \\
0,912 \\
0,900\end{array}$ & $\begin{array}{l}-0,574 \\
-0,662 \\
-0,529 \\
-0,300\end{array}$ & $\begin{array}{l}0,574 \\
0,662 \\
0,529 \\
0,300\end{array}$ & $\begin{array}{l}-2,020 \\
-1,686 \\
-1,588 \\
-2,100\end{array}$ & $\begin{array}{l}-4,040 \\
-3,372 \\
-3,176 \\
-4,200\end{array}$ & $\begin{array}{l}99,87 \\
99,83 \\
99,86 \\
99,94\end{array}$ & $\begin{array}{l}100,13 \\
100,17 \\
100,14 \\
100,06\end{array}$ \\
\hline $\begin{array}{l}\text { 20-34 años } \\
\text { 35-49 años } \\
50 \text { o más }\end{array}$ & $\begin{array}{l}-0,206 \\
-0,704 \\
-0,835\end{array}$ & $\begin{array}{l}0,206 \\
0,704 \\
0,835\end{array}$ & $\begin{array}{l}-0,622 \\
-0,539 \\
-0,671\end{array}$ & $\begin{array}{l}0,622 \\
0,539 \\
0,671\end{array}$ & $\begin{array}{l}-1,433 \\
-1,829 \\
-2,140\end{array}$ & $\begin{array}{l}-2,867 \\
-3,658 \\
-4,280\end{array}$ & $\begin{array}{l}99,81 \\
99,87 \\
99,86\end{array}$ & $\begin{array}{l}100,19 \\
100,13 \\
100,14\end{array}$ \\
\hline $\begin{array}{l}\text { 1-2 integrantes } \\
3-4 \text { integrantes } \\
5 \text { o más int. }\end{array}$ & $\begin{array}{l}-0,787 \\
-0,544 \\
-0,794\end{array}$ & $\begin{array}{l}0,787 \\
0,544 \\
0,794\end{array}$ & $\begin{array}{l}-0,726 \\
-0,563 \\
-0,596\end{array}$ & $\begin{array}{l}0,726 \\
0,563 \\
0,596\end{array}$ & $\begin{array}{l}-1,976 \\
-1,864 \\
-1,787\end{array}$ & $\begin{array}{l}-3,951 \\
-3,727 \\
-3,574\end{array}$ & $\begin{array}{l}99,84 \\
99,87 \\
99,85\end{array}$ & $\begin{array}{l}100,16 \\
100,13 \\
100,15\end{array}$ \\
\hline $\begin{array}{l}\text { Urbana } \\
\text { Rural }\end{array}$ & $\begin{array}{l}-0,639 \\
-0,854\end{array}$ & $\begin{array}{l}0,639 \\
0,854\end{array}$ & $\begin{array}{l}-0,602 \\
-0,875\end{array}$ & $\begin{array}{l}0,602 \\
0,875\end{array}$ & $\begin{array}{l}-1,838 \\
-1,125\end{array}$ & $\begin{array}{l}-3,675 \\
-2,250\end{array}$ & $\begin{array}{l}99,85 \\
99,79\end{array}$ & $\begin{array}{l}100,15 \\
100,21\end{array}$ \\
\hline $\begin{array}{l}\text { Cuenta propia } \\
\text { Empresario } \\
\text { E. particular } \\
\text { E. público } \\
\text { Jubilado } \\
\text { Cesante }\end{array}$ & $\begin{array}{c}-0,789 \\
-0,870 \\
-0,521 \\
-0,457 \\
-0,934 \\
0,750\end{array}$ & $\begin{array}{c}0,789 \\
0,870 \\
0,521 \\
0,457 \\
0,934 \\
-0,750\end{array}$ & $\begin{array}{l}-0,453 \\
-0,700 \\
-0,618 \\
-0,646 \\
-0,580 \\
-1,000\end{array}$ & $\begin{array}{l}0,453 \\
0,700 \\
0,618 \\
0,646 \\
0,580 \\
1,000\end{array}$ & $\begin{array}{l}-1,500 \\
-1,780 \\
-2,035 \\
-1,854 \\
-2,000 \\
-0,500\end{array}$ & $\begin{array}{l}-3,000 \\
-3,560 \\
-4,071 \\
-3,707 \\
-4,000 \\
-1,000\end{array}$ & $\begin{array}{l}99,87 \\
99,83 \\
99,87 \\
99,85 \\
99,87 \\
99,11\end{array}$ & $\begin{array}{l}100,13 \\
100,17 \\
100,13 \\
100,15 \\
100,13 \\
100,89\end{array}$ \\
\hline $\begin{array}{l}\text { Diaria } \\
3 \text { veces/semana } \\
2 \text { veces/semana } \\
\text { Otra frecuencia }\end{array}$ & $\begin{array}{l}-0,653 \\
-0,683 \\
-0,273 \\
-0,867\end{array}$ & $\begin{array}{l}0,653 \\
0,683 \\
0,273 \\
0,867\end{array}$ & $\begin{array}{l}-0,568 \\
-0,679 \\
-0,364 \\
-0,817\end{array}$ & $\begin{array}{l}0,568 \\
0,679 \\
0,364 \\
0,817\end{array}$ & $\begin{array}{l}-1,978 \\
-1,598 \\
-1,909 \\
-1,567\end{array}$ & $\begin{array}{l}-3,956 \\
-3,196 \\
-3,818 \\
-3,133\end{array}$ & $\begin{array}{l}99,50 \\
99,40 \\
99,68 \\
99,27\end{array}$ & $\begin{array}{l}100,50 \\
100,60 \\
100,32 \\
100,73\end{array}$ \\
\hline $\begin{array}{l}\text { \$0-3.999 } \\
\$ 4.000-7.000 \\
\$ 7.001-15.000 \\
\text { Más de } \$ 15.000\end{array}$ & $\begin{array}{c}0,034 \\
-0,870 \\
-0,669 \\
-0,450\end{array}$ & $\begin{array}{l}-0,034 \\
0,870 \\
0,669 \\
0,450\end{array}$ & $\begin{array}{l}-0,635 \\
-0,643 \\
-0,534 \\
-0,350\end{array}$ & $\begin{array}{l}0,635 \\
0,643 \\
0,534 \\
0,350\end{array}$ & $\begin{array}{l}-2,081 \\
-1,513 \\
-2,250 \\
-2,300\end{array}$ & $\begin{array}{l}-4,162 \\
-3,025 \\
-4,500 \\
-4,600\end{array}$ & $\begin{array}{l}99,44 \\
99,43 \\
99,53 \\
99,69\end{array}$ & $\begin{array}{l}100,56 \\
100,57 \\
100,47 \\
100,31\end{array}$ \\
\hline
\end{tabular}

Cifras de utilidad en los distintos niveles de un atributo con signo negativo indican pérdida de utilidad para el consumidor. En el atributo precio, cifras más negativas indican mayor pérdida de utilidad. 
consumidor experimentó utilidad positiva o preferencia frente a la variedad de tomate corriente y negativa respecto a la variedad larga vida, ambas de la misma magnitud pero de signo contrario, resultado que fue similar en la mayoría de las submuestras a excepción de los cesantes y personas que gastan menos de $\$ 4.000$ a la semana en hortalizas, quienes presentaron el comportamiento contrario. Asimismo, tanto en la muestra total como en las submuestras estudiadas los consumidores expresaron utilidad positiva frente al tomate producido en Angol, IX Región, y pérdida de utilidad o rechazo por el tomate de la zona central, lo que concuerda con la distinta evaluación que realizan los consumidores sobre distintos orígenes de los productos determinada por Agrawald y Kamakura (1999), Verlegh y Steenkamp (1999) y Orth \& Firbasová (2003) desde la perspectiva del país de origen y sugerida por Balling (1995) respecto a regiones o provincias. Además, concuerda con la general preferencia de productos nacionales (Kaynak y Cavusgil, 1983; Ettenson et al., 1988; Schnettler et al., 2004), que en esta investigación se refleja en la preferencia por tomate producido en la región de residencia. Además, se encuentra en la misma línea de lo obtenido por Adasme et al. (2006) respecto a la preferencia de frutilla blanca con denominación de origen. Ambos niveles de precio reportaron pérdidas de utilidad en la muestra total y submuestras analizadas, las que se incrementaron al acceder a comprar la hortaliza a un precio mayor $(\$ 300 / \mathrm{kg})$. Esto concuerda con lo determinado por Schnettler et al. (2004) en carne bovina y por Adasme et al. (2006) en frutilla e indica que no existe una asociación entre precio y calidad. En forma congruente con lo anterior, la combinación con mayor proporción de primera preferencia fue tomate corriente producido en Angol a $\$ 150 / \mathrm{kg}(58,9 \%)$, mientras la menos preferida fue tomate larga vida producido en la zona central a \$300/kg (50,6\%).

Disposición a pagar por diferentes orígenes de las hortalizas. En el Cuadro 4 se presenta la DAP-Mag expresada en porcentaje respecto al promedio de los dos niveles de precio utilizados (\$225/ $\mathrm{kg}$ ). Si bien en la muestra total y en las diferentes submuestras analizadas la DAP-Mag por tomates producidos en Angol supera 100\% y la DAP-Mg por tomates de la zona central es inferior a $100 \%$, las diferencias son despreciables. Si se consideran los valores de DAP-Mag del grupo con "otra" frecuencia de compra, que fue el que presentó mayores diferencias al estar dispuestos a pagar 99,27\% del precio promedio de mercado por tomate de la zona central y $0,73 \%$ por sobre el promedio por tomate angolino, traducido a precios implica un precio de $\$ 223 / \mathrm{kg}$ de tomate de la zona central y $\$ 226 / \mathrm{kg}$ de tomate producido en Angol.

Si bien la zona de origen del alimento no presenta la importancia de la procedencia de un producto importado en la decidión de compra, los consumidores de la ciudad de Temuco prefieren tomate producido en la IX Región, aun cuando no están dispuestos a pagar un precio considerablemente superior respecto al mismo producto proveniente de la zona central del país.

\section{LITERATURA CITADA}

ADASME, C.; A. SPILLER Y J. DÍAZ. 2006. Determinación de preferencias del consumidor de la Región Metropolitana hacia la frutilla blanca (Fragaria chiloensis). Un análisis conjunto y una prueba sensorial. Economía Agraria 10: $1-10$.

ADIMARK. 2004. Mapa socioeconómico de Chile. Disponible en http://www.adimark.cl/medios/estudios/informe_mapa socioeconomico de chile.pdf. Conectado el 20 de octubre de 2005 .

AGRAWALD, J. AND W. A. KAMAKURA. 1999. Country of origin: A competitive advantage? International Research in Marketing 16(4): 225-267.

BALESTRINI, P. AND P. GAMBLE. 2006. Country-of-origin effects on Chinese wine consumers. British Food Journal 108(5): 396-412.

BALLING, R. 1995. Der herkunftsaspekt als erfolgsfaktor für das lebensmittelmarketing. Ber Landwirtsch 73: 83-106.
BALTAS, G. AND P. DOYLE. 1998. An empirical analysis of private brand demand recognizing heterogeneous preferences and choice dynamics. Journal of the Operational Research Society 49: 790-798.

BERNUÉS, A.; A. OLAIZOLA, AND K. CORCORAN, K. 2003. Extrinsic attributes of red meat as indicators of quality in Europe: an application for market segmentation. Food Quality and Preference 14: 265-276.

COX, D. N.; A. S. ANDERSON; S. MCKELLAR; J. REYNOLDS; M. E. J. LEAN AND D. J. MELA. 1996. Vegetables and fruit: barriers and opportunities for greater consumption. Nutrition \& Food Science, 96(5): 44-47.

ETTENSON, R.; J. WAGNER AND G. GAETH. 1988. Evaluating the effect of country-of-origin and the "made in USA" campaign: a conjoint approach. Journal of Retailing 64(1) 85-100. 
GAN, C. AND E. LUZAR. 1993. A Conjoint Analysis in Waterfowl Hunting in Louisiana, Journal of Agricultural and Applied Economics 25 (2): 36-45.

GILBERT, L. C. 2000. The functional food trend: what's next and what American think about eggs. Journal of the American College of Nutrition 19(5): 507-512.

HAIR, J.; R. ANDERSON; R. TATHAMY W. BLACK. 1999. Análisis Multivariante. Otero. Quinta edición. Prentice Hall Internacional. Inc. Madrid, España. 832 pp.

IFIC. 2001. Functional foods: can they reduce our risk of cancer? Food insight, May-June.

INE. 1997. Instituto Nacional de Estadísticas. (On line). Quinta encuesta de presupuestos familiares (agosto 1996 - julio 1997). Disponible en: http://www.ine.cl/ine/canales/chile_estadistico/estadisticas_laborales/vencuesta/volumen1/xls/ cuunvoun.xls. Conectado el 10 de enero de 2007.

INE. 1998. (On line). VI Censo Agropecuario, 1997. Disponible en: http://www.ine.cl/canales/chile_estadistico/estadisticas_agropecuarias/xls/2005/'4,04?!A1 Conectado el 10 de enero de 2007.

INE. 2003. Censo 2002. Resultados Volumen I: Población; País - Región. Comisión Nacional del XVII Censo Nacional de Población y VI de Vivienda. Santiago. Chile. 356 pp.

KAYNAK, E. AND S. CAVUSGIL. 1983. Consumer attitudes towards products of foreign origin: Do they vary across product classes? International Journal of Advertising 2(2): 147-157.

LEATHER, S. 1995. Fruit and vegetables: consumption patterns and health consequences. British Food Journal 97(7): 10-17.

LEVIN, R. Y D. RUBIN. 1996. Estadística para Administradores. Prentice Hall, Hispanoamericana S.A. Sexta edición. Juárez. México. 1018 pp.

NICKLAUS, S.; V. BOGGIO; C. CHABANET AND S. ISSANCHOU. 2004. A prospective study of food preferences in childhood. Food Quality and Preference 15: 805-818.

ODEPA. 2006. Oficina de Estudios y Políticas Agrarias (On line). Indicaciones geográficas y denominaciones de origen para productos silvoagropecuarios y agroindustriales. Disponible en: http://www.odepa.gob.cl/odepaweb/servlet/contenidos. ServletDetallesScr;jsessionid=0C7E36993FF3CE46FF5B 693F6E98C9AD?idcla=2\&idn=1782. Conectado el 10 de enero de 2007.

ORTH, U.AND Z. FIRBASOVÁ. 2003. The role of consumer ethnocentrism in food product evaluation. Agribusiness 19(2): 137-153.

SCHEAFFER, R.; W. MENDWENHALL Y L. OTT. 1996. Elementos de Muestreo. Grupo Editorial Iberoamericana S.A. Primera edición. México. 321 pp.

SCHNETTLER, B.; O. MANQUILEF AND H. MIRANDA. 2004. Atributos valorados en la selección de carne bovina en supermercados de Temuco, IX Región de Chile. Ciencia e Investigación Agraria 31(2): 91-100.

VAN ITTERSUM, K.; M. CANDEL AND M. MEULENBERG. 2003. The influence of the image of a product' region of origin on product evaluation. Journal of Business Research 56: 215-226.

VERBEKE, W.; R. WARD AND J. VIAENE. 2000. Probit Analysis of fresh meat consumption in Belgium: exploring BSE and television communication impact. Agribusiness 16(2): 215-234.

VERDUME, A. AND VIAENE, J. 2003. Consumer beliefs and attitude towards genetically modified food: basis for segmentation and implications for communication. Agribusiness, 219 (1): 91-113.

VERLEGH, P. W. J. AND J.-B. E. M STEENKAMP. 1999. A review and meta-analysis of country-of-origin research. Journal of Economic Psychology 20(5): 521-546.

WORLD HEALTH ORGANIZATION (WHO) STUDY GROUP. 1990. Diet, nutrition and the prevention of chronic disease. WHO Technical Reports Series 797. Geneva: World Health Organization.

WHO. 2002. Reducing risks, promoting healthy lives. WHO Technical Reports. Geneva: World Health Organization.

WHO/FAO. 2003. Diet, nutrition and the prevention of chronic diseases. WHO Technical Reports. Geneva: World Health Organization. 